\title{
Performance of Malaria Volunteers regarding Malaria Control Activities in Southeastern Myanmar: A Study in the Areas under Coverage of an Ethnic Health Organization
}

\author{
Ye Thiha, ${ }^{1}$ Thant Zin, ${ }^{1}$ Kyawt Mon Win, ${ }^{2}$ Myat Thu Soe, ${ }^{3}$ and Pyae Linn Aung $\mathbb{D}^{3}$ \\ ${ }^{1}$ STI Myanmar University Campus, University of Bedfordshire (UK), Yangon, Myanmar \\ ${ }^{2}$ Department of Public Health, Ministry of Health and Sports, Naypyitaw, Myanmar \\ ${ }^{3}$ Myanmar Health Network Organization, Yangon, Myanmar \\ Correspondence should be addressed to Pyae Linn Aung; pyaelinnag@gmail.com
}

Received 14 October 2020; Revised 18 December 2020; Accepted 22 December 2020; Published 15 January 2021

Academic Editor: Jianbing $\mathrm{Mu}$

Copyright ( 2021 Ye Thiha et al. This is an open access article distributed under the Creative Commons Attribution License, which permits unrestricted use, distribution, and reproduction in any medium, provided the original work is properly cited.

Malaria volunteers (MVs) play an essential role in resolving malaria problems by delivering greater access to diagnosis and treatment services, mainly for the underserved community residing in hard-to-reach rural areas. The Karen Department of Health and Welfare (KDHW) has implemented community-based malaria control activities among the ethnic minorities in southeastern Myanmar by promoting the roles of MVs. This study aimed to explore the factors influencing the performance of MVs regarding malaria control activities in the area. From July to August 2019, a cross-sectional study was conducted in 12 townships of southeastern Myanmar under the umbrella of the KDHW malaria project. A total of 140 MVs were employed as study participants. Data were collected through face-to-face interviews using a structured questionnaire. For data analyses, descriptive statistics, chi-squared tests, and logistics regression models were applied. More than half of the MVs perceived a good level of performance on malaria control activities. A higher level of performance has been observed among the MVs who had another job (AOR: 1.9, 95\% CI: 1.2-3.9), those experienced in health-related fields (AOR: 1.9, 95\% CI: 1.4-4.9), who received good community support (AOR: 2.1, 95\% CI: 1.3-10.9), who were volunteers beyond three years (AOR: 4.0, 95\% CI: 2.8-9.2), and whose family income totaled over 500,000 MMK (AOR: 2.8, 95\% CI: 1.6-4.2). The results mentioned the characteristics which should be prioritized in recruiting MVs. MV network and their workforce need to be nurtured by encouraging community support. For performance sustainability, attractive incentive schemes or a salary should be subsidized in support of their livelihoods.

\section{Background}

Malaria remains one of the public health problems in many countries, although being a curable and preventable disease. The World Malaria Report estimated 228 million malaria cases and 405,000 malaria deaths occurred globally during 2018 [1]. In Myanmar, malaria remained endemic in 291 out of 330 townships, and more than $60 \%$ of the total population were at risk of malaria infection [2]. Although there was remarkable progress in reducing malaria morbidity and mortality over the last decade, about 75,000 malaria cases and 19 malaria-related deaths were reported in 2018. This situation represented Myanmar as one of the most malaria endemic countries in the Greater Mekong Subregion [1].
Most of the reported malaria cases emerged from areas that were hard to reach in nature, occupied with poor transportation infrastructures, internal armed conflicts, or at cross-border points [3]. Health status in these locations appears to be struggling to improve and the outputs of implemented disease control services were very minimal.

Nevertheless, Myanmar has set a goal of being malaria transmission-free by 2030, with the encouragement of the World Health Organization (WHO) and the country's government [3, 4]. Thus, the National Malaria Control Program (NMCP) has set specific policies in the National Malaria Elimination Plan 2016-2030 [3], including achieving equity in access to services irrespective of gender, race, and ethnicity, especially for the most vulnerable and 
hard-to-reach populations. As part of this, the NMCP has implemented extensive malaria case surveillance systems at the village level by supporting the village volunteers' workforce. Currently, NMCP has trained and supported more than 15,000 active malaria volunteers (MVs) over the country in collaboration with nongovernment organizations, international partners, and Ethnic Health Organizations (EHOs) $[2,5]$. A malaria volunteer refers to a person residing in hard-to-reach areas where no government health facilities exist and is performing health services voluntarily to the local community without pay [6]. In 2016, those MVs performed diagnosis and treatment for 73,404 malaria cases, accounting for nearly $70 \%$ of the country's achievement in community case management $[2,6]$. That figure means the participation of MVs in Myanmar has been playing a major role in ensuring universal access to malaria diagnosis and treatment among hard-to-reach and at-risk populations (including mobile and migrants populations, forest-related workers usually residing in remote, cross-border, and ethnic groups), especially in rural areas.

The WHO has highlighted that early diagnosis and treatment serves as the most effective way to prevent an uncomplicated malaria case from developing into more severe conditions or death [7,8]. This strategy was also important for eradicating the gametocide stage in the case of Plasmodium falciparum infection to interrupt possible onward transmission [7]. In an area with massive malaria caseloads, increased access to diagnosis and treatment facilities through MV posts would be an effective solution to reduce the disease burden within a reasonable time frame [9]. A systematic literature review also pointed out the importance of community health volunteers in implementing effective community case management for malaria [10]. As such, ensuring MVs' performance in optimum and regular needs assessment is important to conduct day-to-day malaria prevention and control activities smoothly and to reach country-wide universal health coverage. A study concluded that strengthening of knowledge and attitudes, the presence of family support, and delivering other motivational incentives acted as necessary components to create better roles of MVs in malaria control activities [11].

In an area with ongoing political instability and conflicts, malaria control services were suggested to be impossible to deliver through the government sector. For an intervention or activity, it might be critical to enter into a local community unless the involvement of local people from a particular location already existed [12]. For this reason, EHOs were found in the 1900s for the sake of health for ethnic minorities residing in conflict-affected areas which were mostly peripheral to the government health care services [13]. The EHOs trained and supported village MVs chosen from the local people. The organizations also distributed diagnostic tools and antimalarial drugs for MVs to serve as the frontline malaria service providers. Thus the capacity of EHOs should be properly strengthened for those isolated areas to sustain the progress towards the national malaria elimination target. Additionally, health care promotions in these postconflict areas would also strengthen the national reconciliation process. Therefore, this study aimed to evaluate the performance of MVs and its influences among the volunteers who provide malaria control activities among Kayin ethnic people under the coverage of an EHO in Myanmar.

\section{Methods}

2.1. Study Area. The study was purposely conducted in 12 townships of Southeastern Myanmar where the Regional Artemisinin Resistance Initiative to Elimination (RAI-2E) malaria project through the Global Fund has been implemented by the Karen Department of Health and Welfare (KDHW), one of the EHOs in Myanmar. These 12 townships included Shwegyin, Kyaukkyi, Hpa-an, Kawkareik, Myawaddy, Kyarinseikgyi, Thaton, Billin, Kyaikhto, Ye, Tanintharyi, and Bokpyin (Figure 1). All these townships were recorded as hard-to-reach areas according to road accessibility across seasons assessed by the General Administration Department [14]. These locations were selected to conduct the research due to the unique geographical nature of the areas and the presence of similar population characteristics. The townships were known for being malaria endemic, remote, and where the capacity of the health care providers including the MVs regarding malaria control activities had not yet been analyzed. Furthermore, the area served as a malaria hot spot due to the enormous cross-migration of the population being the border areas of Thailand and Myanmar.

The KDHW had trained and supported an average of 25 volunteers in each township, totaling 290 volunteers, to provide malaria diagnosis and treatment among hard-toreach and at-risk populations especially Kayin ethnic people of rural communities from 12 townships of southeastern Myanmar. Generally, four criteria to select the villages were usually used to deploy a volunteer [5]: (i) village with high malaria burden, (ii) hard-to-reach location, (iii) village with relatively high population density, and (iv) village where no health care facilities or health staff close by. Next, to select a MV $[5,6]$, the preferred individuals should be (i) a permanent resident of a village, (ii) accepted by the community, (iii) willing to work as volunteers, (iv) male or female with 18 years to 50 years of age, and (v) able to read and write Myanmar language and speak local dialects. In addition, recommendations from respective village administrative groups were also considered while recruiting the volunteers. Moreover, overlapping with other similar projects was avoided.

After choosing a volunteer, the organization provided a five-day recruitment training for malaria case management and reporting mechanisms. After training, the volunteers were allowed to use rapid diagnostic test (RDT) (SD BIOLINE Malaria $\operatorname{Ag} P . f / P . v)$ kits and to treat uncomplicated malaria cases according to the national malaria treatment guidelines. Volunteers were encouraged to refer the cases (complicated malaria, malaria with pregnancy, or $<1$-yr-old children). The key activities of volunteers included but were not limited to (i) providing early diagnosis and treatment of uncomplicated malaria cases, (ii) referring severe and complicated malaria patients to the nearest hospital or health facilities, (iii) 


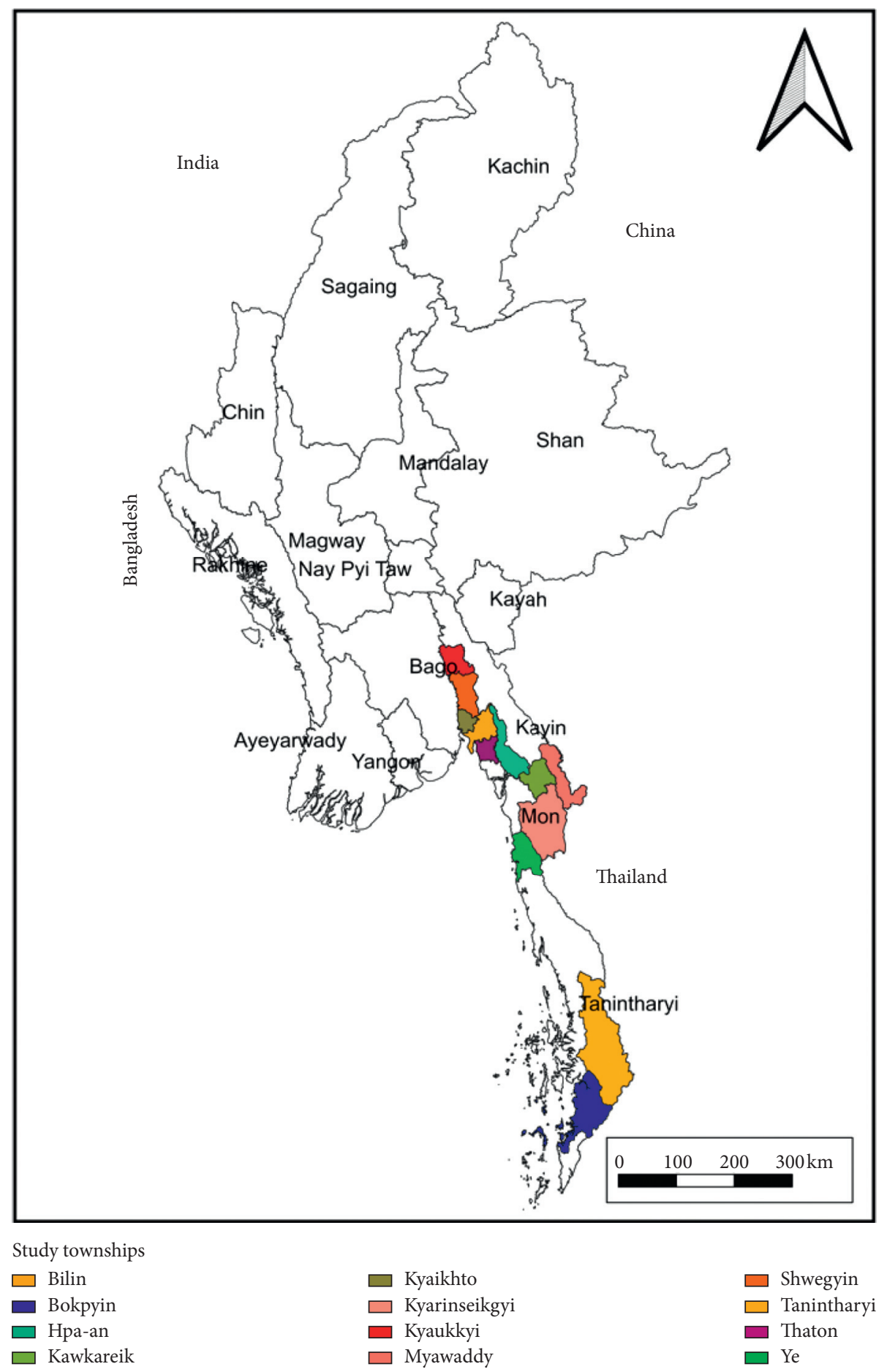

FIGURE 1: Study townships.

distributing materials like pamphlets, posters, and longlasting insecticide-treated nets (LLINs) to community people, (iv) educating people to promote malaria prevention and control, (v) observing proper LLIN use by the community, and (vi) recording and reporting of patients credentials. Routine on-the-job training as well as annual refresher courses were also organized.
The KDHW supported the incentives, either monetary ( 15USD monthly) or nonmonetary rewards such as umbrellas, backpacks, hats, and torch lights, for the volunteers depending on the instruction of the national malaria control program or budget availability. The organization also delivered monthly supportive monitoring and evaluation visits by field or central supervisors to ensure the quality of malaria 
care services as well as data recording and reporting and to solve any issues or challenges faced by the assigned volunteers.

2.2. Sampling Procedures. The 290 volunteers, who had completed the training and were providing malaria health care services to the community in the 12 selected townships under the supervision of the KDHW for at least six months, were eligible to participate in this study. However, those refusing to participate in the interview and those unable to communicate well due to serious illness were excluded from the study. The sample size was determined by using Epi Info ${ }^{\mathrm{TM}}-7$ Software by putting the proportion of volunteers who revealed the good performances in Myanmar at $44.0 \%$, as stated in one study [11]. Then the required sample size should be at least 140 by adding $10 \%$ more for the dropout and nonrespondent rate. A list of eligible volunteers separated by township was requested from the KDHW. To achieve the desired sample size, an average of 12 volunteers from each township was selected using systematic random sampling through a number lottery method.

2.3. Study Tools. The study deployed primary data collection through a quantitative cross-sectional design using a structured questionnaire. The questionnaire was adapted from previous relevant studies $[11,15]$ and another tool [16]. It was firstly developed in English and then translated to Myanmar language by the research team. The questionnaire was divided into six parts described below.

Part I: sociodemographic factors of the respondents - the nine questions included age in years, sex either male or female, marital status, educational level, types of occupation, average annual family income, related working experience, and duration as volunteer. The answers were predefined as ministatements and listed under each question.

Part II: knowledge-this part consisted of 13 miniquestions regarding procedures of RDT testing, treatment guidelines, and information on LLINs. The questions consisted of both positive and negative statements. The answers could be "yes," "no," or "do not know." For the correct answer, the respondents received " 1 " score but received " 0 " score if they gave an incorrect answer or responded as "do not know."

Part III: perception of roles and functions of volunteer-the six questions included both positive and negative structures. By using a three-point Likert's rating scale [17], the scores were given in the order of " 3 ," " 2 ," and " 1 " for "agree," "uncertain," and "disagree," respectively, for positive statements. Reciprocally, the scores of " 1 ," " 2 ," and " 3 " were assigned for "agree," "uncertain," and "disagree" in case of negative statements.

Part IV: family support-this section included four questions. Only one choice was allowed, and the scoring criteria were always $=3$, sometimes $=2$, and never $=1$ for positive questions or always $=1$, sometimes $=2$, and never $=3$ for negative questions.

Part V: community support-this part was constructed using four questions and the answers included "always," "sometimes," and "never" according to the three-point Likert's scale model. The scores were formulated as " 3 " for "always," " 2 " for "sometimes," and “1” for "never," accordingly.

Part VI: performance of the volunteers-this was defined as the volunteers insights upon their current practices regarding malaria control activities guided by the organization. The activities were firstly constructed based on the village volunteer algorithm endorsed by the national program [6]. Some activities were narrowed down and skipped upon considering several relevant studies and reports $[6,11,15,16,18]$ and also their reliability in the actual field situation. Finally, 11 activities represented as the key activities of a MV which overall addressed performing blood testing, prescribing of antimalarial medicines, operating health education sessions, delivering preventive measures, and the last for data recording and reporting data. The answers were structured as a three-point Likert's scale using "always," "sometimes," and "never." The scoring system (always $=3$, sometimes $=2$, never $=1$ ) was applied.

The overall scores of the level of knowledge level, perception level, family and community support, and performance were compiled and categorized into two groups using Bloom's Taxonomy theory [19] as $<80$ of total score $=$ poor level and $\geq 80 \%$ of total score $=$ good level.

2.4. Data Collection. Two research assistants were hired among the local residents who attained at least a bachelor's degree and fluency in local language and tones. They were trained for two days about the nature of the study including the flow of the questionnaire, data collection process, and research ethics in conducting study involving human subjects. Practical sessions were also demonstrated to reflect the real situation and to be aware of the interviewing time. In this study, data were collected at the township level during the quarterly coordination meetings conducted by KDHW during July and August 2019. In the early morning before meeting time, each selected volunteer's name was announced and requested to receive an interview. Two research assistants conducted face-to-face interviews with the validated questionnaires in a private room away from other people. The interviewing environment was also secured from other people entering to avoid any annoyance. An interview normally took not more than 30 minutes.

2.5. Data Processing and Analysis. The consistency, completeness, and integrity of the data were checked immediately by the researchers after collecting data daily. Data were encoded and entered into EpiData software. After that, all the data were transferred and analyzed using Statistical Package for the Social Sciences (SPSS, version 23). 
Frequencies, proportions, means, and standard deviations for descriptive analysis were used to describe sociodemographic factors, knowledge, perception of volunteers regarding malaria, family and community support, and the performance of volunteers. To determine significant differences between dependent and independent variables, bivariate analysis using chi-squared tests were applied. To obtain absolute chances for study variables and avoid including too many redundant variables, the variables which showed $p$ value $<0.05$ during chi-squared tests were analyzed in simple and multiple logistics regression models to demonstrate the odd ratios and $95 \%$ confidence intervals.

\section{Results}

3.1. Sociodemographic Characteristics of the MVs. This study deployed a total of 140 malaria volunteers. The ages of the volunteers ranged from 18 to 50 years. The majority (71.5\%) was under 30 years. About two-thirds were married female. Most participants attained secondary school and lower level education. For occupation, 32.1\% worked in an agricultural related occupation followed by owning a business (28.6\%) and the daily wages laborers (20.0\%). Many (62.1\%) earned more than 500,000 MMK as annual family income ranging from 150,000 MMK as minimum and 1,800,000 MMK as maximum. Most respondents (65.7\%) did not posses no working experience of health-related work before being a MV. Regarding the time of being a volunteer, more than $81 \%$ of respondents worked as a volunteer for more than one year ranging from one to six years (Table 1).

\subsection{Knowledge about Malaria Control Activities.} Regarding knowledge on current using the malaria RDT, almost all the study's respondents correctly answered about the storage of malaria RDT at the coolest place where the temperature was less than $40^{\circ} \mathrm{C}$, including the drops of buffer solution to be placed in the RDT. However, only 75.0 and $85.7 \%$ of malaria volunteers gave the right answers on the time frame for reading results and concluding the results based on the bends appearing in the RDT, respectively. For questions concerning prescription antimalarial drugs, all respondents knew well about referring severe or complicated malaria cases to the nearest hospitals or health care facility. More than $90 \%$ of volunteers delivered the correct dosage schedule to treat $P$. falciparum, $P$. vivax and mixed infections accordingly. However, some often prescribed antimalarial medicines among patients with negative RDT results. Moreover, more than $99 \%$ of respondents knew that using LLINs was the most effective way to prevent mosquito bites and that sleeping under LLIN was the best way to prevent malaria. Next, more than $92 \%$ were aware that the effectiveness of LLINs exceeded beyond one year. Interestingly, more than $70 \%$ believed that LLINs could cause skin itchiness and respiratory tract infection, particularly among children (Table 2 ).

3.3. Perceptions towards Roles and Functions of the MVs. Among the statements addressing the perceptions of the studies volunteers towards their roles and functions, all respondents agreed with the importance of timely referral
TABLE 1: Sociodemographic characteristics of the 140 respondents.

\begin{tabular}{|c|c|c|}
\hline Characteristics & $n$ & $\%$ \\
\hline \multicolumn{3}{|l|}{ Age (years) } \\
\hline $18-30$ & 100 & 71.5 \\
\hline $31-40$ & 24 & 17.1 \\
\hline$>41$ & 16 & 11.4 \\
\hline Mean \pm SD & \multicolumn{2}{|c|}{$28.2 \pm 8.9$} \\
\hline Min-Max & \multicolumn{2}{|c|}{$18-50$} \\
\hline \multicolumn{3}{|l|}{ Sex } \\
\hline Male & 57 & 40.7 \\
\hline Female & 83 & 59.3 \\
\hline \multicolumn{3}{|l|}{ Marital status } \\
\hline Single & 53 & 37.9 \\
\hline Married & 87 & 62.1 \\
\hline \multicolumn{3}{|l|}{ Education } \\
\hline Primary school & 44 & 31.4 \\
\hline Secondary school & 48 & 34.3 \\
\hline High school & 42 & 30.0 \\
\hline College/Bachelor & 1 & 0.7 \\
\hline $\begin{array}{l}\text { Others (monastery } \\
\text { education/illiterate) }\end{array}$ & 5 & 3.6 \\
\hline \multicolumn{3}{|c|}{ Major occupation (apart from malaria volunteer work) } \\
\hline Unemployed & 6 & 4.3 \\
\hline Agriculture/farmers & 45 & 32.1 \\
\hline Own business & 40 & 28.6 \\
\hline Dependent/housewives & 21 & 15.0 \\
\hline Daily wages labors & 28 & 20.0 \\
\hline \multicolumn{3}{|c|}{ Annual family income $(M M K)^{*}$} \\
\hline$\leq 500,000$ & 53 & 37.9 \\
\hline$>500,000$ & 87 & 62.1 \\
\hline Mean \pm SD & $87,0071 \pm 95$ & \\
\hline Min-Max & $150,000-1,8$ & \\
\hline \multicolumn{3}{|c|}{$\begin{array}{l}\text { Working experience related to health before being a } \\
\text { malaria volunteer }\end{array}$} \\
\hline Ever & 48 & 34.3 \\
\hline Never & 92 & 65.7 \\
\hline \multicolumn{3}{|c|}{ Time of being a malaria volunteer (year) } \\
\hline$\leq 1$ & 25 & 17.9 \\
\hline $1-3$ & 69 & 49.3 \\
\hline$>3$ & 46 & 32.8 \\
\hline Mean \pm S.D & $1.80 \pm$ & \\
\hline Min-Max & 0.5 & \\
\hline
\end{tabular}

* 1,300 MMK 1 USD, SD: standard deviation, Min: minimum, and Max: maximum.

for severe or complicated malaria cases to receive life saving measures, building good communication mechanisms within community was crucial and the last, they served essential roles in delivering malaria control activities and preventing the emergence of drug resistant malaria. Further, $13.6 \%$ of the respondents did not realize that the disseminating of health knowledge was a necessary tool for malaria prevention and control. Next, $29.3 \%$ disagreed that timely submission of records and reports was important. Last, $22.9 \%$ thought that the assessment on the proper use of LLINs in the community was unnecessary (Table 3 ).

3.4. Family and Community Support for the MVs. As shown in Table 4, most respondents (82.1\%) always received kind 
TABLE 2: Correct knowledge about malaria control activities $(n=140)$.

\begin{tabular}{|c|c|c|}
\hline \multirow{2}{*}{ Knowledge statements } & \multicolumn{2}{|c|}{ Correct answer } \\
\hline & $n$ & $\%$ \\
\hline \multicolumn{3}{|l|}{ Malaria rapid diagnostic test (RDT) } \\
\hline Keep RDTs in cool place of your residence (within $1-40^{\circ} \mathrm{C}$ ) & 135 & 96.4 \\
\hline${ }^{*}$ Put 8 drops of buffer solution into the buffer well perpendicularly & 140 & 100 \\
\hline Read RDT's results within $15-30$ minutes & 105 & 75.0 \\
\hline If RDT appeared lines at C\&P. $f$, the result is $P$. falciparum & 120 & 85.7 \\
\hline \multicolumn{3}{|l|}{ Prescribing antimalarial medicines } \\
\hline P. falciparum-artemisinin combination therapy (ACT) (3 days) + primaquine (stat on first day) & 133 & 95.0 \\
\hline P. vivax-chloroquine ( 3 days) + primaquine (weekly for 8 consecutive weeks) & 134 & 95.7 \\
\hline Mixed infection-ACT ( 3 days) + primaquine (weekly for 8 consecutive weeks) & 131 & 93.6 \\
\hline${ }^{*} \mathrm{RDT}(-)$ ve patients-antimalarial medicine should be prescribed & 136 & 97.1 \\
\hline Severe or complicated malaria-referral & 140 & 100 \\
\hline \multicolumn{3}{|l|}{ Long lasting insecticide treated nets (LLINs) } \\
\hline Using LLINs is the most effective way to prevent mosquito bites. & 139 & 99.3 \\
\hline Sleeping under LLIN is the best way to prevent malaria. & 139 & 99.3 \\
\hline *Sleeping under LLIN can cause skin itchiness and respiratory tract infection among children & 38 & 27.1 \\
\hline${ }^{*}$ LLIN can be effective for one year only & 129 & 92.1 \\
\hline
\end{tabular}

${ }^{*}$ Negative questions; RDT: rapid diagnostic test.

TABLe 3: Perceptions towards roles and functions of malaria volunteers $(n=140)$.

\begin{tabular}{|c|c|c|c|c|c|c|}
\hline \multirow[t]{2}{*}{ Statements } & \multicolumn{2}{|c|}{ Agree } & \multicolumn{2}{|c|}{ Disagree } & \multicolumn{2}{|c|}{ Uncertain } \\
\hline & $n$ & $\%$ & $n$ & $\%$ & $N$ & $\%$ \\
\hline $\begin{array}{l}\text { Giving knowledge on malaria to local people individually and in a } \\
\text { group is a necessary tool for the prevention and control of malaria }\end{array}$ & 121 & 86.4 & 19 & 13.6 & 0 & 0 \\
\hline Timely submission of patients records and reports is important & 99 & 70.7 & 41 & 29.3 & 0 & 0 \\
\hline Timely referral for severe malaria cases is essential for life saving & 140 & 100 & 0 & 0 & 0 & 0 \\
\hline It is necessary to build good communication with community & 140 & 100 & 0 & 0 & 0 & 0 \\
\hline $\begin{array}{l}\text { Malaria volunteers play an important role in implementing malaria } \\
\text { control activities and prevention of drug resistant malaria }\end{array}$ & 140 & 100 & 0 & 0 & 0 & 0 \\
\hline${ }^{*}$ Monitoring the proper use of LLINs in the community should not be done & 32 & 22.9 & 104 & 74.3 & 4 & 2.8 \\
\hline
\end{tabular}

${ }^{*}$ Negative statements.

encouragements from their family members to work as a MV. However, $45.7 \%$ of respondents revealed their family members sometimes accompanied them in implementing activities. About 93\% of the respondents never received complaints from family members on decreased time spent with the family and lower family income because of the volunteer service. Regarding community supports, $80 \%$ of the respondents received encouragement from the villagers to work as a malaria volunteer. Around two-thirds of the participants also described the community support regarding the aspects of providing information on new suspected malaria cases, active participation in malaria control activities, and following treatment procedures and preventive methods.

\subsection{Performance of MVs regarding Malaria Control Activities.} Performance of MVs regarding malaria diagnosis was assessed. Most respondents (82.1\%) performed RDT testing for every malaria-suspected case. When handling test kits,
93.6\% followed the procedures. The volunteers also strictly followed the malaria treatment guidelines. Most (98.6\%) provided the instructions for how to take the medicines as well. More than 99\% usually referred to every severe or complicated malaria cases to higher level facilities. Despite $89.3 \%$ admitting that they delivered the health messages while waiting for the RDT result for 15 minutes, some (23.6\%) did not distribute the education aids materials like pamphlets and posters. More than $87 \%$ alluded that they were always involved in LLINs distribution. Almost all the volunteers (98.6\%) also carefully recorded the required information in the detailed Case Report Forms (CRFs). However, many participants (30.7\%) had never observed the LLIN use by the community. All the respondents submitted the records and reports according to the targeted time frame, either by month or by quarter (Table 5).

3.6. Overall Levels of Knowledge, Attitude, Family Support, Community Support, and Performance of MVs. Table 6 
TABLE 4: Family support and community support for the malaria volunteer $(n=140)$.

\begin{tabular}{|c|c|c|c|c|c|c|}
\hline \multirow{2}{*}{ Descriptions } & \multicolumn{2}{|c|}{ Always } & \multicolumn{2}{|c|}{ Sometimes } & \multicolumn{2}{|c|}{ Never } \\
\hline & $n$ & $\%$ & $n$ & $\%$ & $n$ & $\%$ \\
\hline \multicolumn{7}{|l|}{ Family support } \\
\hline Members of your family encourage you to voluntarily work as a malaria volunteer & 115 & 82.1 & 23 & 16.4 & 2 & 1.5 \\
\hline Members of your family join you in your activities & 61 & 43.6 & 64 & 45.7 & 15 & 10.7 \\
\hline${ }^{*}$ Working as a volunteer, your family complains of lesser family income & 10 & 7.1 & 0 & 0 & 130 & 92.9 \\
\hline${ }^{*}$ Working as a volunteer, your family complains about decreased time spent on them & 8 & 5.7 & 2 & 1.4 & 130 & 92.9 \\
\hline \multicolumn{7}{|l|}{ Community support } \\
\hline People in your village encourage you to voluntarily work as a malaria volunteer & 112 & 80.0 & 19 & 13.6 & 9 & 6.4 \\
\hline People in your village informed you for the new suspected cases of malaria & 96 & 68.6 & 35 & 25.0 & 9 & 6.4 \\
\hline People in your village actively participated in all of your activities & 87 & 62.2 & 45 & 32.1 & 8 & 5.7 \\
\hline People in your village adhered on malaria treatment, prevention, and control guidelines & 95 & 67.9 & 41 & 29.3 & 4 & 2.8 \\
\hline
\end{tabular}

${ }^{*}$ Negative statements.

Table 5: Performance of 140 respondents regarding malaria control activities.

\begin{tabular}{|c|c|c|c|c|c|c|}
\hline \multirow{2}{*}{ Activities } & \multicolumn{2}{|c|}{ Always } & \multicolumn{2}{|c|}{ Sometimes } & \multicolumn{2}{|c|}{ Never } \\
\hline & $N$ & $(\%)$ & $N$ & $(\%)$ & $N$ & $(\%)$ \\
\hline \multicolumn{7}{|l|}{ Malaria diagnosis } \\
\hline Diagnose every suspected cases of malaria with RDT & 115 & 82.1 & 25 & 17.9 & 0 & 0 \\
\hline Strictly follow the procedure of RDT testing & 131 & 93.6 & 9 & 6.4 & 0 & 0 \\
\hline \multicolumn{7}{|l|}{ Malaria treatment } \\
\hline Follow the guidelines of the malaria treatment & 140 & 100 & 0 & 0 & 0 & 0 \\
\hline Give instruction on full course of malaria drugs & 138 & 98.6 & 2 & 1.4 & 0 & 0 \\
\hline $\begin{array}{l}\text { Refer every severe malaria case to the nearest hospitals/health facilities } \\
\text { Health education }\end{array}$ & 139 & 99.3 & 1 & 0.7 & 0 & 0 \\
\hline $\begin{array}{l}\text { Provide education on malaria control and prevention individually } \\
\text { while waiting for RTD's result }\end{array}$ & 125 & 89.3 & 15 & 10.7 & 0 & 0 \\
\hline $\begin{array}{l}\text { Distribute pamphlets and posters to the RTD tested patients as well as community } \\
\text { Malaria prevention }\end{array}$ & 80 & 57.1 & 33 & 23.6 & 27 & 19.3 \\
\hline Properly distribute LLINs to the community depend on the availability of resources & 122 & 87.1 & 16 & 11.4 & 2 & 1.5 \\
\hline Observe how people use LLINs effectively & 43 & 30.7 & 54 & 38.6 & 43 & 30.7 \\
\hline $\begin{array}{l}\text { Record patients characteristics, RDT result and prescribed medications correctly in CRFs } \\
\text { Data recording and reporting }\end{array}$ & 138 & 98.6 & 2 & 1.4 & 0 & 0 \\
\hline Submit records and reports as scheduled & 139 & 99.3 & 1 & 0.7 & 0 & 0 \\
\hline
\end{tabular}

RDT: rapid diagnostic test, LLIN: long-lasting insecticide-treated net, CRF: case report form.

summarizes the overall levels of knowledge, attitude, family support, community support, and performance of the studied MVs. Two groups for each variable were constructed. Most respondents (85.7\%) showed a good level of knowledge; similarly, $87.1 \%$ expressed positive perception. Regarding family and community support, $72.1 \%$ of respondents received good family support, while $67.1 \%$ maintained good support from the respective community. Additionally, $53.6 \%$ of the respondents perceived good level of performance.

3.7. Relationships between Sociodemographic Factors, Knowledge, Attitude, Family Support, Community Support, and Performance of MVs. The cross tabulations were conducted between the performance of MVs and other variables including sociodemographic characteristics of the respondents, their levels of knowledge, attitude, and receiving support from family and community. The higher the age above 30 years, the greater proportions of good level performance were observed. Female volunteers revealed higher possession of good roles than those of male respondents. Similarly, married MVs who attained above primary level education and possessed a job in addition to a volunteer work and family income of $>500,000 \mathrm{MMK}$ had better performance. Next, those who had previous experience of working in a health related area before being a volunteer and volunteers who worked in this field for more than one year were likely to show better performance. Last, MVs with a background of good community support demonstrated good performance roles (Table 7 ).

The possible relationships were addressed by the chisquared test as shown in Table 7 . These results were insignificant concerning some sociodemographic characteristics of the respondents such as age, sex, marital status, level of education, level of knowledge, level of attitude and family support, and performance of MVs. Further, statistically significant differences were observed among types of occupation in addition to volunteer work $(p=0.002)$, ranges 
TABLE 6: Level of knowledge, level of attitude, family support, community support, and performance of malaria volunteers $(n=140)$.

\begin{tabular}{lcc}
\hline Descriptions & $n$ & $\%$ \\
\hline $\begin{array}{l}\text { Levels of knowledge } \\
\text { Good }\end{array}$ & 120 & 85.7 \\
\hline Need to improve & 20 & 14.3 \\
\hline $\begin{array}{l}\text { Levels of perception } \\
\text { Positive perception }\end{array}$ & 122 & 87.1 \\
\hline Negative perception & 18 & 12.9 \\
\hline $\begin{array}{l}\text { Family support } \\
\text { Good }\end{array}$ & 101 & 72.1 \\
\hline Poor & 39 & 27.9 \\
\hline $\begin{array}{l}\text { Community support } \\
\text { Good }\end{array}$ & 94 & 67.1 \\
\hline Poor & 46 & 32.9 \\
\hline Performance of the malaria volunteer & & \\
Good & 75 & 46.4 \\
\hline Poor & 65 & \\
\hline
\end{tabular}

Grouping by Bloom's Taxonomy theory, good level: $\geq 80 \%$ of the total score; poor level: $<80 \%$ of the total score.

of annual family income $(p=0.010)$, time of being a volunteer $(p=0.034)$, possessing working experience related to health work before serving as a volunteer $(p=0.025)$, and performance levels regarding malaria control activities at $p$-value $<0.05$.

3.8. Logistic Regression Models. Both simple and logistic regression models were applied. In regression analysis, the performance of MVs showed approximately twofold increments among employed MVs in addition to volunteer position (AOR: 1.9, 95\% CI: 1.2-3.9), individuals experienced in a health related field before serving as a volunteer (AOR: 1.9, 95\% CI: 1.4-4.9), and those who received good support from their communities (AOR: 2.1, 95\% CI: 1.3-10.9). Moreover, a longer time being a volunteer served as a backbone for better performance, including MWs with 1 to 3 years' experience (AOR: 1.9, 95\%CI: 1.5-2.9) and MWs with more than 3 years' experience (AOR: 4.0, 95\%CI: 2.8-9.2). Furthermore, the greater odds were also observed among these respondents whose family income totaled more than 500,000 MMK, towards good levels of performance (AOR: 2.8, 95\% CI: 1.6-4.2) (Table 8).

\section{Discussion}

This study investigated the performance of MVs who devoted their lives as frontline malaria service providers especially in rural and remote areas of Myanmar where government health care services were scarcest. The results summarized that more than one-half of the studied MVs provided a good level of performance in implementing malaria control activities. The factors enhancing the roles of the MVs were being employed rather than solely providing volunteer work, having experience in a health related sector before serving as a volunteer, a longer time serving as a volunteer, and having family income totaling over one half million MMK. Nevertheless, other variables such as age, sex, educational attainments, marital status, knowledge levels, attitude levels, and family support suggested only insignificant relationships with $\mathrm{MV}$ performance. The majority population in Myanmar lives below the poverty line and generally cannot access quality health care and encounters high out-of-pocket expenditures [20]. On the contrary, the current health system in Myanmar has been considered fragmented and unstable at the system level [21]. In addition, universal health coverage remains fragile [20]. These continue to threaten the achievement of sustainable development goals for the country. Meanwhile, MVs serve as the first contact point for delivering health services to vulnerable populations by increasing access to diagnosis and treatment facilities. According to one country report, around $70.0 \%$ of patients with malaria were tested and treated by the volunteer workforce [2]. In an area with high malaria caseloads, the burden has been progressively declined after introducing the MVs [9]. Using an integrated approach, the volunteers performed very well for symptomatic treatment of other minor illnesses like seasonal flu and referrals of major infectious diseases like tuberculosis and HIV [22]. However, the roles of volunteers should be sustained once malaria problems decreased, mainly during the final stages of the malaria elimination era. Otherwise, malaria elimination might not be attained by the targeted timeline of 2030 . Therefore, understanding the factors which might influence the performance of the volunteers would have a certain advantage in strengthening the roles of volunteers in the upcoming elimination trajectory.

MVs were mainly responsible for routine case surveillance using the passive case detection approach, i.e., when a patient with malaria-like symptoms sought treatment at the volunteer's post, they provided a blood test and prescribed treatment as necessary. Malaria is highly seasonal and the trend was usually high during rainy seasons (July-October) [23]. Therefore, before or after malaria peak seasons, the volunteers generally had less workload and did not need to contribute full-time volunteer work unless their respective 
TABLE 7: Relationships between sociodemographic factors, knowledge, attitude, family support, community support, and performance level of malaria volunteer $(n=140)$.

\begin{tabular}{|c|c|c|c|c|c|}
\hline \multirow{3}{*}{ Descriptions } & \multicolumn{4}{|c|}{ Level of performance } & \multirow{3}{*}{$p$ value } \\
\hline & \multicolumn{2}{|c|}{ Good } & \multicolumn{2}{|c|}{ Poor } & \\
\hline & $n$ & $\%$ & $n$ & $\%$ & \\
\hline \multicolumn{6}{|l|}{ Age (years) } \\
\hline $18-30$ & 52 & 52.0 & 48 & 48.0 & \multirow{3}{*}{0.56} \\
\hline $31-40$ & 13 & 54.2 & 11 & 45.8 & \\
\hline$>41$ & 10 & 62.5 & 6 & 37.5 & \\
\hline \multicolumn{6}{|l|}{ Gender } \\
\hline Male & 27 & 47.4 & 30 & 52.6 & \multirow{2}{*}{0.22} \\
\hline Female & 48 & 57.8 & 35 & 42.2 & \\
\hline \multicolumn{6}{|l|}{ Marital status } \\
\hline Single & 25 & 47.2 & 28 & 52.8 & \multirow{2}{*}{0.23} \\
\hline Married & 50 & 57.5 & 37 & 42.5 & \\
\hline \multicolumn{6}{|l|}{ Education } \\
\hline Primary and below & 23 & 46.9 & 26 & 53.1 & \multirow{2}{*}{0.24} \\
\hline Above primary & 52 & 57.1 & 39 & 42.9 & \\
\hline \multicolumn{6}{|c|}{ Major occupation (apart from volunteer work) } \\
\hline Unemployed & 4 & 66.7 & 2 & 33.3 & \multirow{2}{*}{$0.002 *$} \\
\hline With job & 71 & 53.0 & 63 & 47.0 & \\
\hline \multicolumn{6}{|c|}{ Annual family income $(M M K) * *$} \\
\hline$\leq 500,000$ & 21 & 39.6 & 32 & 60.4 & \multirow{2}{*}{$0.010^{*}$} \\
\hline$>50,0000$ & 54 & 62.1 & 33 & 37.9 & \\
\hline \multicolumn{6}{|c|}{ Working experience related to health before being as a volunteer } \\
\hline Ever $\quad 1$ in & 32 & 66.7 & 16 & 33.3 & \multirow{2}{*}{$0.025^{*}$} \\
\hline Never & 43 & 46.7 & 49 & 53.3 & \\
\hline \multicolumn{6}{|c|}{ Time period of being as a volunteer (year) } \\
\hline$\leq 1$ & 10 & 40.0 & 15 & 60.0 & \multirow{3}{*}{$0.034^{*}$} \\
\hline $1-3$ & 37 & 53.6 & 32 & 46.4 & \\
\hline \multirow{2}{*}{\multicolumn{6}{|c|}{ Knowledge level }} \\
\hline & & & & & \\
\hline Good & 66 & 55.0 & 54 & 45.0 & \multirow{2}{*}{0.406} \\
\hline Need to improve & 9 & 45.0 & 11 & 55.0 & \\
\hline \multicolumn{6}{|l|}{ Attitude level } \\
\hline Positive perception & 67 & 54.9 & 55 & 45.1 & \multirow{2}{*}{0.281} \\
\hline Negative perception & 8 & 44.4 & 10 & 55.6 & \\
\hline \multicolumn{6}{|l|}{ Family support } \\
\hline Good & 59 & 58.4 & 42 & 41.6 & \\
\hline Poor & 16 & 41.0 & 23 & 59.0 & 0.064 \\
\hline Community support & & & & & \\
\hline Good & 60 & 63.8 & 34 & 36.2 & \\
\hline Poor & 15 & 32.6 & 31 & 67.4 & $0.001^{*}$ \\
\hline
\end{tabular}

${ }^{*}{ }^{*} 1,300 \mathrm{MMK} \sim 1$ USD; degree of freedom for chi-square test $=1 ;{ }^{*}$ significance at $p<0.05$.

organizations had assigned other duties. A volunteer could easily spare some time to work in another job especially to increase family income. It might also reduce the production of complaints by other family members concerning lower income or less family bonding time because of volunteer work. Other studies have confirmed a similar finding that volunteers with another source of income or a paid job tended to show good performance $[11,24]$. In a populated village or an area with very high malaria risks, the recruitment of more than one volunteer would be more efficient. Instead of organizing frequent township level meetings or training, on-site training during monitoring visits was recommended. However, a well-designed backup plan should be in place to substitute the low performance volunteers who were busy with nonvolunteer work and failed in routine reporting or showed zero reporting for some consecutive months without strong justification.

MVs voluntarily performed malaria-related health services in the community without pay. However, the organization routinely supported monthly incentives in line with the guidelines from the national program. Yet, the MVs were also satisfied with the current amount of payment. Nevertheless, the result of this study suggested that the volunteers who retained higher family income were likely to exhibit greater performance levels. Of course, a person working as a volunteer without pay might experience stress for the wellbeing of other members. Once family members have established a backup for gross family income, the volunteer could focus on malaria control activities for the sake of improving community health. A study in Myanmar also 
TABLE 8: Influencing factors for the performance of malaria volunteers $(n=140)$.

\begin{tabular}{|c|c|c|c|c|}
\hline Descriptions & COR & $95 \% \mathrm{CI}$ & AOR & $95 \% \mathrm{CI}$ \\
\hline \multicolumn{5}{|c|}{ Major occupation (apart from volunteer work) } \\
\hline Unemployed & 1 & - & 1 & - \\
\hline With job & 3.2 & $(2.1-4.2)$ & 1.9 & $(1.2-3.9)$ \\
\hline \multicolumn{5}{|c|}{ Annual family income $(M M K)^{*}$} \\
\hline$\leq 500,000$ & 1 & - & 1 & - \\
\hline$>500,000$ & 2.9 & $(2.0-4.6)$ & 2.8 & $(1.6-4.2)$ \\
\hline \multicolumn{5}{|c|}{ Working experience related to health before being a malaria volunteer } \\
\hline Never & 1 & - & 1 & - \\
\hline Ever & 2.4 & $(1.8-4.5)$ & 1.9 & $(1.5-2.9)$ \\
\hline \multicolumn{5}{|c|}{ Time of being a malaria volunteer (year) } \\
\hline$\leq 1$ & 1 & - & 1 & - \\
\hline $1-3$ & 2.6 & $(1.8-5.2)$ & 1.9 & $(1.4-4.9)$ \\
\hline$>3$ & 4.1 & $(3.1-9.2)$ & 4.0 & $(2.8-9.2)$ \\
\hline \multicolumn{5}{|c|}{ Community support } \\
\hline Poor & 1 & - & 1 & - \\
\hline Good & 2.9 & $(1.4-11.2)$ & 2.1 & $(1.3-10.9)$ \\
\hline
\end{tabular}

*1,300 MMK 1 USD, COR: crude odds ratio by simple logistic regression, AOR: adjusted odds ratio by multiple logistic regression, and CI: confidence interval.

formulated the unique fact that volunteers with high family income delivered good roles in implementing malaria control activities [11]. Because malaria programs are vertical in nature, depending on under which auspice they are in, the level of support and type of support they received from their organizations might differ according to the donor's policies, budget allowance, and organizational rules. The national program should endorse a standardized rate that can entirely cover the efforts made by the volunteers. Whenever possible, this rate should reflect the minimal daily wage of a citizen (i.e., 7,000 MMK/day), approved by the Ministry of Labor [25]. Alternatively, when selecting volunteers, their family income should be informally assessed.

The MVs having health related working experience showed better roles than those volunteers without health related working experience. The finding was similar to that of another study conducting a country-wide survey in Myanmar [11]. Local people certainly realized a person who was very active in the community worked with underlying better health knowledge than other villagers. Therefore, they usually pointed out that person to help or be involved in health related activities organized by various organizations. Subsequently, $34.3 \%$ of studied participants possessed health related working experience. Those individuals were aware of the nature of health interventions and demonstrated good communication skills regarding health care projects and organizations. They might receive job-related training as well. These experiences might enhance the volunteers in absorbing the knowledge transferred during the training and also helped them to easily follow the diagnostic procedures [26]. Individuals possessing health related experience should be given precedence in being selected as a volunteer. However, recruiting a volunteer by many organizations should be precluded to avoid works overload, data repetition, and time conflicts using a different scope of assigned work.

Generally, a person who decided to commit to a single job for a longer time would then gradually become more skillful than that an individual who frequently changed their positions at different work places. The underlying reasons included but were not limited to underlying enthusiastic mindsets, attending relevant training, receiving job-related experiences, and sharing knowledge among colleagues and mentorships from immediate supervisors. Likewise, in this study, the volunteers with more than one year's experience represented better roles in implementing malaria control activities. A similar result could be observed in another study in which volunteers with longer experience showed more appropriate roles in their performance [11]. The longer the time of being a volunteer, the more people recognized the performance of volunteers and appreciated the existence of MVs within the community in controlling and preventing deadly diseases [27]. The appreciation from the respective community would be another reason for MVs to perform good roles. To offset the low attrition rate among trained MVs, each organization might want to implement an award system for higher performing volunteers. Introducing an output-based incentive scheme under proper supervision might also be a solution. In the case volunteers moved to another location though they still wanted to participate as a volunteer, their names should be referred to the organization that operates in the new location.

Whenever village-based health interventions were delivered, the MVs were critical to operating activities smoothly. Given that fewer human resources were available in the health sector, support from the villagers was usually encouraged to accelerate the activities. Similarly, the work of volunteers could not succeed without proper cooperation from the community, mainly for 
adhering to treatment procedures, following preventive measures especially the use of bed nets, seeking diagnostic testing in time, and attending health education sessions [28]. In this study, the volunteers with good community support performed better than those receiving poor support. The finding was supported by a study conducted in Myanmar and Malawi $[29,30]$. Once the volunteers received the respect of the community, they would strengthen future activities. The respective organization should advocate the community members and village stakeholders about the roles of volunteers in the villages and offer support by explaining the importance of community involvement in malaria control activities delivered by the MVs. Additionally, some activities like organizing awareness-raising campaigns, LLINs distribution, and surveying its use should be accompanied by higher level officials together with the MVs.

Because this constituted a cross-sectional quantitative study, like any survey, any causal relationships between influencing factors and the performance status of malaria volunteers were unable to confirm. A qualitative interview may be warranted to understand the contextual factors behind their performance. As the responses were self-reported, to minimize any bias, it would be better to introduce other validation steps in accessing their roles, for example, validate their past records, treatment compliance, reporting regularity, testing performances, and other performance benchmarks, and then triangulate with their knowledge, skills, and perceptions. This study only assessed MVs participating under the KDHW. Thus, the ability to generalize the findings would be limited as this study could not provide any findings from different organizations that delivered different award systems, program designs, monitoring and reporting systems, incentives, and supervisors although each organization might implement uniform treatment guidelines, diagnosis, and referral system. The finding was also subject to bias in particular concerning assessing the community members' participation and family members' support which were subjective and comprised just the opinions of the volunteers. The volunteers might be reluctant to openly disclose about collaborating with village leaders and the armed groups due to fear of dismissal, as such findings on high community participation level might have been biased. Due to the quantitative nature of the study, providing an in-depth understanding of the problem is impossible.

\section{Conclusion}

The study summarized those characteristics which should be prioritized in future recruitments of MVs. MVs networks and their workforce need to be nurtured by encouraging community support. To eliminate malaria, indisputably, these VHV networks and workforce need to be strengthened as only these mechanism exist to ensure universal coverage of malaria services in all underserved communities across the country. Current MVs are unengaged as employees recognized by any health system (either government or NGO), but rather are supported as volunteers and only minor compensation was made as recognition of the time they devoted to delivering health services. Regarding performance sustainability, attractive incentive schemes or a salary should be subsidized to support their livelihoods. However, the current incentives were still perceived as good motivational factors for the MVs, so that the national program needs to review the appropriateness of such schemes while balancing the economy, value for money, and sustainability. Last, "health constitutes a bridge for peace," supporting the health of the community in nongovernment controlled areas representing the door to further continue the ongoing peace building process in Myanmar.
Abbreviations
AOR: Adjusted odds ratio
CI: Confidence interval
COR: Crude odds ratio
CRF: $\quad$ Case report form
EHO: Ethnic Health Organization
KDHW: Karen Department of Health and Welfare
LLIN: Long-lasting insecticide-treated net
MVs: Malaria volunteers
NGO: Nongovernment organization
NMCP: National Malaria Control Program
RAI: Regional artemisinin resistance initiative
RDT: Rapid diagnostic test
SPSS: Statistical Package for the Social Sciences
WHO: World Health Organization.

\section{Data Availability}

All the data analyzed for this study are included within the article.

\section{Ethical Approval}

The researchers obtained ethical approval from the Institutional Review Board of Defense Services Medical Research Center (DSMRC) as well as the Institute for Health Research Ethics Committee (IHREC) of the University of Bedfordshire (UOB) before commencing the research.

\section{Conflicts of Interest}

The authors declare they have no conflicts of interest.

\section{Authors' Contributions}

YTH and TZ conceived the design of this study. YTH, TZ, and KMW facilitated data collection. YTH, MTS, and PLA performed analysis and drafted the manuscript. All authors reviewed and revised the manuscript and read and approved the final manuscript.

\section{Acknowledgments}

The authors would like to thank Dr. Ed Marta for granting approval to collect data for this study in KDHW's operating areas. 


\section{References}

[1] World Health Organization, World Malaria Report 2019, WHO, Geneva, Switzerland, 2019.

[2] Department of Public Health, Ministry of Health and Sports, National Strategic Plan: Intensifying Malaria Control and Accelerating Progress towards Malaria Elimination (20162020), Ministry of Health and Sports, Naypyidaw, Myanmar, 2016.

[3] Department of Public Health, Ministry of Health and Sports, National Plan for Malaria Elimination in Myanmar (20162030), Ministry of Health and Sports, Naypyidaw, Myanmar, 2016.

[4] World Health Organization, Global Technical Strategy for Malaria 2016-2030, WHO, Geneva, Switzerland, 2015.

[5] Ohnmar, M. Tun, S. San, W. Than, and V. Chongsuvivatwong, "Effects of malaria volunteer training on coverage and timeliness of diagnosis: a cluster randomized controlled trial in Myanmar," Malaria Journal, vol. 11, p. 309, 2012.

[6] Department of Public Health, Ministry of Health and Sports, Manual for Intergrated Community Malaria Volunteer, Ministry of Health and Sports, Naypyidaw, Myanmar, 2017.

[7] World Health Organization, Overview of Malaria Treatment, WHO, Geneva, Switzerland, 2018.

[8] National Malaria Control Program, Department of Public Health, World Health Organization, Updated National Malaria Treatment Guidelines 2015, MOHS, National Malaria Control Program, Department of Public Health, World Health Organization, Naypyitaw, Myanmar, 2015.

[9] J. Landier, D. M. Parker, A. M. Thu et al., "The role of early detection and treatment in malaria elimination," Malaria Journal, vol. 15, p. 363, 2016.

[10] L. Smith Paintain, B. Willey, S. Kedenge et al., "Community health workers and stand-alone or integrated case management of malaria: a systematic literature review," The American Journal of Tropical Medicine and Hygiene, vol. 91, no. 3, pp. 461-470, 2014.

[11] P. L. Aung, T. Silawan, T. Rawiworrakul, and M. Min, "Perceived role and its enhancing factors among the village health volunteers regarding malaria control in rural Myanmar," Indian Journal of Public Health, vol. 62, no. 1, pp. 10-14, 2018.

[12] K. Baltzell, K. Harvard, M. Hanley, R. Gosling, and I. Chen, "What is community engagement and how can it drive malaria elimination? case studies and stakeholder interviews," Malaria Journal, vol. 18, no. 1, p. 245, 2019.

[13] Mae Tao Clinic About Us (Mae Tao Clinic): https:// maetaoclinic.org/about-us/history/.

[14] Myanmar Information Management Unit, Hard to Reach Areas (Maps and Shapefiles). Yangon, Myanmar, MIMU, Yangon, Myanmar, 2019, http://themimu.info/news/hardreach-areas-maps-and-shapefiles.

[15] M. H. Nyunt, K. M. Aye, K. T. Kyaw et al., "Challenges encountered by local health volunteers in early diagnosis and prompt treatment of malaria in Myanmar artemisinin resistance containment zones," Malaria Journa, vol. 15, p. 308, 2016.

[16] Myanmar National Malaria Control Programme, Myanmar Malaria Indicator Survey, NMCP, Naypyitaw, Myanmar, 2015.

[17] H. N. Boone and D. A. Boone, "Analyzing likert data," Journal of Extension, vol. 50, no. 2, pp. 1-5, 2012.

[18] S. E. Canavati, S. Lawpoolsri, C. E. Quintero et al., "Village malaria worker performance key to the elimination of artemisinin-resistant malaria: a Western Cambodia health system assessment," Malaria Journal, vol. 15, no. 1, p. 282, 2016.

[19] C. Stanny, "Reevaluating bloom's taxonomy: what measurable verbs can and cannot say about student learning," Education Sciences, vol. 6, no. 4, p. 37, 2016.

[20] A. Ergo, T. S. Htoo, R. Badiani-Magnusson, and R. Royono, "A new hope: from neglect of the health sector to aspirations for universal health coverage in Myanmar," Health Policy and Planning, vol. 34, no. 1, pp. i38-i46, 2019.

[21] M. Van Rooijen, C. Y. Myint, M. Pavlova, and W. Groot, "Health insurance in Myanmar: the views and perception of healthcare consumers and health system informants on the establishment of a nationwide health insurance system," Risks, vol. 6, no. 3, p. 81, 2018.

[22] A. R. D. McLean, H. P. Wai, A. M. Thu et al., "Malaria elimination in remote communities requires integration of malaria control activities into general health care: an observational study and interrupted time series analysis in Myanmar," BMC Medical, vol. 16, no. 1, p. 183, 2018.

[23] J. Geng, P. Malla, J. Zhang et al., "Increasing trends of malaria in a border area of the greater mekong subregion," Malaria Journal, vol. 18, no. 1, p. 309, 2019.

[24] K. Alfes, B. Antunes, and A. D. Shantz, "The management of volunteers-what can human resources do? a review and research agenda," The International Journal of Human Resource Management, vol. 28, no. 1, pp. 62-97, 2017.

[25] S. Campbell, "Labour formalisation as selective hegemony in Reform-era Myanmar," The Asia Pacific Journal of Anthropology, vol. 20, no. 1, pp. 57-73, 2019.

[26] J. D. Rae, S. Nosten, S. Proux et al., "The role of monitoring and evaluation to ensure functional access to communitybased early diagnosis and treatment in a malaria elimination programme in Eastern Myanmar," Malaria Journal, vol. 18, no. 1, p. 50, 2019.

[27] N. Y. Y. Linn, S. Kathirvel, M. Das et al., "Are village health volunteers as good as basic health staffs in providing malaria care? a country wide analysis from Myanmar 2015," Malaria Journal, vol. 17, no. 1, Article ID 242, 2018.

[28] K. Shahandeh and H. R. Basseri, "Challenges and the path forward on malaria elimination intervention: a systematic review," Iranian Journal of Public Health, vol. 48, no. 6, pp. 1004-1013, 2019.

[29] N. Y. Y. Linn, J. P. Tripathy, T. M. Maung et al., "How are the village health volunteers deliver malaria testing and treatment services and what are the challenges they are facing? a mixed methods study in Myanmar," Tropical Medicine and Health, vol. 46, p. 28, 2018.

[30] B. N. Kaunda-Khangamwa, H. van den Berg, R. S. McCann et al., "The role of health animators in malaria control: a qualitative study of the health animator (HA) approach within the majete malaria project (MMP) in chikwawa district, Malawi," BMC Health Services Research, vol. 19, no. 1, p. 478, 2019. 